\title{
Effects of Oral Rinse using Lemon-Flavored Water with or without Rebamipide on Fluoropyrimidine-Induced Stomatitis
}

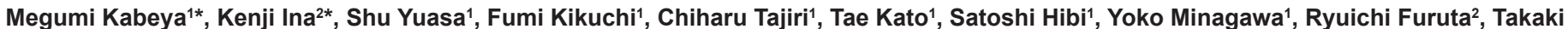
Kikuchi $^{3}$, Takashi Kanamori ${ }^{3}$, Satoshi Kayukawa ${ }^{3}$, and Takae Kataoka ${ }^{3}$

${ }^{1}$ Department of Hospital Pharmacy, Nagoya Memorial Hospital, Nagoya, Japan

${ }^{2}$ Department of Medical Oncology, Nagoya Memorial Hospital, Nagoya, Japan

${ }^{3}$ Department of Clinical Oncology, Nagoya Memorial Hospital, Nagoya, Japan

\section{Abstract}

Aim: To examine the efficacy of oral rinsing against chemotherapy-induced stomatitis.

Methods: Consecutive fluoropyrimidine-treated patients with stomach and colorectal cancer were enrolled from April 2009 to March 2011 ( $n=43$; Group 1) and from January 2012 to December 2012 ( $n=45$; Group 2). The incidence and severity of stomatitis were compared between Group 1 patients, who were instructed to gargle with lemonflavored water 6 times daily, and Group 2 patients, who did not receive any specific guidance. Among patients in the gargle group, we determined the rate of gargling compliance as well as quality of life (QOL) scores, and evaluated the impact of rebamipide use on patient outcomes.

Results: The incidence of stomatitis was significantly reduced in Group 1 (14.0\%) compared to that in Group $2(33.3 \%)$ and its severity in the former group was milder. Among patients using lemon-flavored water, concomitant rebamipide use had no statistically significant impact on stomatitis incidence (with versus without rebamipide, $19.0 \%$ versus $9.0 \%$ ), the rate of gargling compliance $(96.4 \%$ versus $94.2 \%)$, or QOL scores.

Conclusions: Oral rinse with lemon-flavored water is useful for the symptomatic control of fluoropyrimidineinduced stomatitis, regardless of the presence or absence of rebamipide.

Keywords: Oral rinsing; Stomatitis; Quality of life; Fluoropyrimidines; Rebamipide

\section{Introduction}

In Japan, stomach and colorectal cancers are the most frequent causes of cancer-related death [1-3] despite dramatic advances in diagnosis and surgical treatment. Chemotherapy plays an important role in the treatment of these cancers, and fluoropyrimidines, such as 5-fluorouracil (5-FU) and S-1, are widely used as key drugs [4,5]. Furthermore, the recent progress in chemotherapy has extended the overall survival of patients with these cancers $[2,3,6]$, in whom fluoropyrimidines are sometimes used for periods longer than a year. Oral mucositis is one of the most common symptomatic complications associated with the use of fluoropyrimidines $[7,8]$. Severe oral mucositis can cause intolerable pain and increase the risk of systemic infections, necessitating dose reduction and discontinuation of cancer chemotherapy. Moreover, this adverse effect may have an impact on patient nutrition and quality of life (QOL) [9], and therefore, an effective and prophylactic intervention to alleviate this complication is needed.

The Multinational Association for Supportive Care/International Society of Oral Oncology mucositis guidelines for cancer patients recommend maintaining good oral hygiene, emphasizing the importance of keeping the oral cavity clean $[8,10]$. Moisture in the oral cavity may alleviate irritation caused by mechanical contact between the teeth and oral mucosa, and oral dryness may exacerbate oral mucositis. Therefore, keeping the oral cavity moist is important for the prevention of stomatitis. Some studies have suggested that anti-ulcer agents may be effective for the symptomatic control of oral mucositis in cancer patients $[11,12]$. Rebamipide, one of the cytoprotective agents originally used for the treatment of gastroduodenal ulcers [13], is well known to increase mucus secretion [14]. Moreover, rebamipide has also been suggested to attenuate mucosal injury through the suppression of oxidative stress and reactive oxygen species (ROS) production and through the exertion of inhibitory effects on pro-inflammatory cytokines, such as tumor necrosis factor-alpha (TNF- $\alpha$ ) and interleukins 1 and $6[13,15]$.

We examined the clinical efficacy of oral rinse with lemon-flavored water, with or without rebamipide, against oral mucositis during the 8 weeks following chemotherapy initiation. Because of its strong and bitter aftertaste, $100 \%$ Pokka Lemon ${ }^{\circledR}$ (Pokka Sapporo Food \& Beverage Ltd., Sapporo, Japan) flavoring was used with the rebamipide solution [16]. We then compared the clinical effects of oral rinse with lemonflavored water, alone or with rebamipide, on the incidence and degree of stomatitis, the rate of gargling compliance, and QOL scores.

\section{Materials and Methods}

\section{Patients}

Patients were eligible for this study if they signed an informed consent document and met all of the following criteria: (1) pathologically proven gastric or colorectal cancer; (2) age 20-85 years; (3) Eastern Cooperative Oncology Group performance status of 0-2; (4) WBC count between 4,000 and $12,000 / \mathrm{mm}^{3}$, platelet count greater than $100,000 / \mathrm{mm}^{3}$, hemoglobin greater than $8 \mathrm{~g} / \mathrm{dL}$, and serum

*Corresponding author: Kenji Ina, Department of Medical Oncology, Nagoya Memorial Hospital, 4-305 Hirabari, Tenpaku-ku, Nagoya 468-8520, Japan, Tel: +81-52-804-1111; Fax: +81-52-803-8830; E-mail: kina@hospy.or.jp

Received August 06, 2013; Accepted September 07, 2013; Published September 17, 2013

Citation: Kabeya M, Ina K, Yuasa S, Kikuchi F, Tajiri C, et al. (2013) Effects of Oral Rinse using Lemon-Flavored Water with or without Rebamipide on Fluoropyrimidine-Induced Stomatitis. J Integr Oncol 2: 107. doi:10.4172/23296771.1000107

Copyright: (c) 2013 Kabeya M, et al. This is an open-access article distributed under the terms of the Creative Commons Attribution License, which permits unrestricted use, distribution, and reproduction in any medium, provided the original author and source are credited. 
Citation: Kabeya M, Ina K, Yuasa S, Kikuchi F, Tajiri C, et al. (2013) Effects of Oral Rinse using Lemon-Flavored Water with or without Rebamipide on Fluoropyrimidine-Induced Stomatitis. J Integr Oncol 2: 107. doi:10.4172/2329-6771.1000107

Page 2 of 4

bilirubin less than $1.5 \mathrm{mg} / \mathrm{dL}$; and (5) receiving fluoropyrimidines for chemotherapy. Between April 2009 and March 2011, 48 consecutive eligible patients were enrolled. Among them, 5 patients were excluded because their follow-up did not reach 8 weeks, mainly due to changes in or discontinuation of the protocol because of disease progression. The remaining 43 patients (Group 1) were assigned into one of 2 treatment groups: Group 1A patients received lemon-flavored water alone, and Group 1B patients received lemon-flavored water containing rebamipide. A second group of 50 consecutive patients (gastric cancer; 25 and colorectal cancer; 25) who met the same criteria were enrolled from January 2012 to December 2012. From these, 5 patients were excluded because changes to their treatment protocol were made within 8 weeks, and 45 patients who received no specific instruction regarding oral rinsing were examined (Group 2). This study was approved by the institutional review board of Nagoya Memorial Hospital.

\section{Oral care}

Group 1 patients were instructed to gargle 6 times per day (after each meal, at 10:00 AM, at 3:00 PM, and before sleep) for 8 weeks with $300 \mathrm{~mL} /$ day of water containing $5-10 \mathrm{~mL}$ of $100 \%$ Pokka Lemon ${ }^{\mathbb{B}}$ with or without $300 \mathrm{mg} /$ day rebamipide and followed by oral rinsing with $50 \mathrm{ml}$ of tap water [17-19]. They were also instructed on daily gargle solution preparation by a multidisciplinary team using an instruction sheet (Figure 1). Pokka Lemon ${ }^{\circledR}$ juice is commercially available at a reasonable price (costing 483 yen for 8 weeks) and is easy to prepare. As previously described [16], the content of rebamipide in an acidic solution with a $\mathrm{PH}$ of 3-4 was $99.3 \%$ after $4 \mathrm{~h}$ and $98.4 \%$ after $8 \mathrm{~h}$, indicating sufficient stability in lemon-flavored water. Therefore, Pokka Lemon $^{\circledR}$ was chosen as the flavoring agent for the rebamipide gargle solution used in this prospective study. To determine compliance rates, patients were instructed to use a diary and self-check during the 8-week treatment period. Meanwhile, the comparison patients in Group 2 were explained about the adverse effects of fluoropyrimidine treatment without receiving any specific instruction on oral rinsing.

\section{Assessment of oral mucositis and QOL}

Oral mucositis was diagnosed by a multidisciplinary team for the duration of chemotherapy. Its severity in patients receiving fluoropyrimidine-based chemotherapy (Groups 1 and 2) was evaluated by several operators (Ina K, Furuta R, Kikuchi F, Tajiri C, and Kato T)

\section{Guide for the gargle}

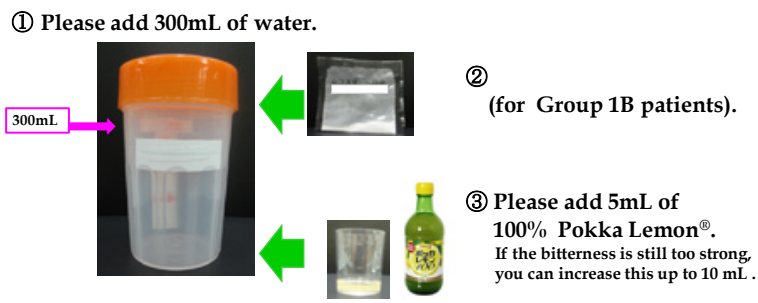

(4) Please shake well to dissolve the solution.

(5) Please gargle with $50 \mathrm{~mL}$ of the mixed solution once.

(6) Please gargle with $50 \mathrm{~mL}$ of tap water.

O Please gargle 6 times per day (after each meal, at 10:00 AM, at 03:00 PM, and before sleep), and use all of the solution in 1 day.

O Please shake well to dissolve the solution before each gargle.

$\mathrm{O}$ The bitterness may remain after gargling ; however, please do not drink or eat for 30 minutes.

Figure 1: Instruction sheet of gargle used in this study.

\begin{tabular}{|c|c|c|}
\hline & Group 1 & Group2 \\
\hline Period & April 2009-March 2011 & $\begin{array}{c}\text { January } 2012-\text { December } \\
2012\end{array}$ \\
\hline Number & 43 & 45 \\
\hline \multicolumn{3}{|l|}{ Gender } \\
\hline Male & 29 & 34 \\
\hline Female & 14 & 11 \\
\hline \multicolumn{3}{|l|}{ Age (years) } \\
\hline Median & 63 & 69 \\
\hline Range & $41-81$ & $50-84$ \\
\hline \multicolumn{3}{|l|}{ Primary site } \\
\hline Colorectal & 24 & 23 \\
\hline Stomach & 19 & 22 \\
\hline
\end{tabular}

Table 1: Characteristics of patients in Groups 1 and 2.

through physical examination and careful interview according to the National Cancer Institute Common Terminology Criteria for Adverse Events (NCI-CTCAE) version 4.0. In Group 1, evaluation of QOL was performed on days 1, 28, and 56 using 2 questionnaires: the European Organization for Research and Treatment of Cancer (EORTC) QLQ-C-30 and the General Oral Health Assessment Index (GOHAI) [20,21]. The EORTC QLQ-C-30 comprises 30 questions, incorporating 5 functional scales (physical, role, cognitive, emotional, and social functioning), 3 symptom scales (fatigue, pain, and nausea), several single-item symptom measures, and a global health and QOL scale. The GOHAI questionnaire was originally developed to assess oral health problems in older adults. This questionnaire is also a self-reported assessment of QOL containing 12 questions. Studies have suggested that the GOHAI questionnaire is more sensitive to relatively mild oral symptoms compared with other indices, such as the Oral Impacts on Daily Performance index $[22,23]$.

\section{Statistical analysis}

Frequencies of oral mucositis were compared between the 2 groups using Fisher's test. Comparison of stomatitis severity was performed using the Mann-Whitney U test. EORTC QLQ-C-30 and GOHAI scores were compared on days 1,28 , and 56 using the Bonferroni test. Differences in compliance rates and QOL scores between Groups 1A and $1 \mathrm{~B}$ were analyzed using the Mann-Whitney $\mathrm{U}$ test. $P$-values were calculated using StatFlex statistical software (Artech, Osaka, Japan), and a P-value of less than 0.05 was considered significant.

\section{Results}

Group 1 included 43 fluoropyrimidine-treated patients with stomach and colorectal cancers (19 cases versus 24 cases) who were enrolled during the earlier enrollment period, while Group 2 included 45 similar patients (stomach cancer; 22 cases versus colorectal cancer; 23 cases) who were enrolled during the later enrollment period. Patient characteristics for both groups are shown in Table 1 . The 8-week incidence of oral mucositis was significantly lower in Group 1 than in Group 2 (14.0\% versus 33.3\%; $P=0.0454)$. Within 8 weeks, 6 Group 1 patients (14.0\%) suffered from grade 1 oral mucositis, whereas in Group 2 there were 7 cases of grade 1 oral mucositis (15.6\%), 5 cases of grade 2 oral mucositis (11.1\%), and 3 cases of grade 3 oral mucositis $(6.7 \%)$ (Figure 2). The severity of oral mucositis also differed significantly between the 2 groups $(P=0.0178)$.

Of the 43 patients in Group 1, 22 were assigned to Group 1A (without rebamipide) and 21 were assigned to Group 1B (with rebamipide) (Table 2). There were no statistically significant differences in the incidence of stomatitis between these groups ( $1 \mathrm{~A}$ versus $1 \mathrm{~B}, 9.0 \%$ versus 


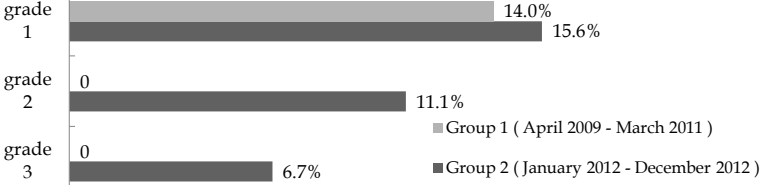

$P=0.0178$ (Group 1 versus Group 2) using the Mann-Whitney $U$ test $P$-values were calculated using StatFlex statistical software (Artech, Osaka, Japan), and a $P$-value of less than 0.05 was considered significant.

Figure 2: Severity of oral mucositis according to NCI-CTCAE version 4.0

\begin{tabular}{|c|c|c|}
\hline & $\begin{array}{c}\text { Group 1A } \\
\begin{array}{c}\text { (without rebamipide) } \\
(n=22)\end{array}\end{array}$ & $\begin{array}{c}\text { Group 1B } \\
\text { (with rebamipide) } \\
(n=21)\end{array}$ \\
\hline \multicolumn{3}{|l|}{ Gender } \\
\hline Male & 14 & 15 \\
\hline Female & 8 & 6 \\
\hline \multicolumn{3}{|l|}{ Age (year) } \\
\hline Median & 62 & 68 \\
\hline Range & $41-79$ & $57-81$ \\
\hline \multicolumn{3}{|l|}{ Performance status } \\
\hline 0 & 10 & 14 \\
\hline 1 & 11 & 6 \\
\hline 2 & 1 & 1 \\
\hline \multicolumn{3}{|l|}{ Primary tumor site } \\
\hline Colorectal & 13 & 11 \\
\hline Stomach & 9 & 10 \\
\hline \multicolumn{3}{|l|}{ Chemotherapy } \\
\hline S-1 alone & 2 & 4 \\
\hline S-1/paclitaxel & 3 & 3 \\
\hline S-1/cisplatin & 2 & 2 \\
\hline PSC & 1 & 1 \\
\hline UFT alone & 1 & \\
\hline UFT/LV & & 1 \\
\hline mFOLFOX6 & 4 & 2 \\
\hline FOLFIRI+bevacizumab & 2 & 2 \\
\hline FOLFIRI+cetuximab & 1 & 1 \\
\hline XELOX & 3 & 2 \\
\hline Capecitabine alone & 2 & 2 \\
\hline XELOX+bevacizumab & 1 & 1 \\
\hline
\end{tabular}

PSC: Paclitaxel plus S-1/cisplatin combination therapy [32]; mFOLFOX: intravenous bolus and infusional 5 -fluorouracil/folinic plus oxaliplatin; FOLFIRI: intravenous bolus and infusional 5-fluorouracil/folinic plus irinotecan; XELOX: capecitabine plus oxaliplatin

Table 2: Characteristics of patients randomly assigned into the 2 groups.

$19.0 \%)$. Fairly good rates of gargling compliance were achieved in both Groups 1A (94.2\%) and 1B (96.4\%), indicating that nearly all Group 1 patients were able to complete oral rinsing 6 times daily. QOL scores using the EORTC QLQ-C-30 were chronologically determined for each patient at days 1,28 , and 56 after the initiation of chemotherapy. All 30 measures of this QOL assessment showed no significant changes from before to after chemotherapy in both Groups 1A and 1B. Evaluation of serial changes in GOHAI scores for both groups as determined at day $1(53.3 \pm 7.8$ versus $56.0 \pm 5.5$, respectively), day $28(55.9 \pm 12.7$ versus $53.5 \pm 13.5$, respectively), and day $56(56.8 \pm 4.7$ versus $53.8 \pm$ 6.1 , respectively) revealed essentially the same tendency as those of the EORTC QLQ-C-30. Both questionnaires indicated that the presence of rebamipide treatment did not impact QOL at each time point.

\section{Discussion}

In the current study, oral mucositis was investigated in patients with stomach and colorectal cancer receiving fluoropyrimidine-based chemotherapy. We first determined the incidence and severity of oral mucositis in the 8 weeks following chemotherapy initiation and compared these between 2 patient groups enrolled at different periods: April 2009 to March 2011 (Group 1) and January 2012 to December 2012 (Group 2). We found that patients in the earlier enrollment group, who were instructed to gargle 6 times a day, suffered from oral mucositis less frequently than patients in the second enrollment group, who were not specifically instructed to gargle. Recent randomized studies in patients with gastrointestinal cancer showed that the incidence of stomatitis was $25-51 \%$ in those receiving 5-FU-based chemotherapy $[5,24,25]$. The incidence of oral mucositis in Group $2(15 / 45 ; 33.3 \%)$ was comparable to that in previous reports, whereas the incidence in Group $1(6 / 43 ; 14.0 \%)$ was significantly lower. In addition, the degree of oral mucositis in Group 1 was exclusively assessed as grade 1, while in Group 2 the severity of oral mucositis ranged from grade 1 to grade 3. These differences in the incidence and severity of stomatitis between Groups 1 and 2 should support the efficacy of oral rinsing with lemonflavored water. Oral mucositis may lead to a reduction in the response to chemotherapy as a result of treatment interruption or inadequate treatment doses [26-28]. As fluoropyrimidine-based chemotherapy has been widely used in the treatment of gastrointestinal cancer, the development of an effective and prophylactic intervention to alleviate oral mucositis is warranted. Because there is currently no specific therapy to control this complication, the importance of oral rinsing with lemon-flavored water cannot be over-exaggerated.

We failed to detect any additional effects of rebamipide when added to a lemon-flavored water oral rinse with regard to compliance or QOL. This study does not have enough power to detect any differences between patients gargling with lemon-flavored water alone and those gargling with lemon-flavored water containing rebamipide, because Group 1 comprised only 43 patients in total. However, we still place emphasis on that the severity of stomatitis was mild in patients, who were instructed to gargle 6 times daily. These findings may imply that oral rinsing with lemon-flavored water is effective for the alleviation of stomatitis and maintenance of QOL, regardless of the presence or absence of rebamipide in the gargle solution.

Group 1 patients were more frequent recipients of a multidisciplinary approach to oral care than those in Group 2, as they were instructed on the gargle preparation and procedure. Because we have neither dentists nor dental hygienists at our institution, our study saw active contributions from pharmacists in collaboration with the nursing staff, who provided patients with instructions on gargle preparation and conducting self-checks using the diary in adition to administering 2 types of QOL questionnaires. Frequent communication with members of the multidisciplinary team enhanced patient compliance with the gargle regimen, which may be very helpful in the prophylactic intervention of oral mucositis. Microorganisms present in the saliva and oral cavity have potential roles in mucosal inflammation [29]. Because oral streptococci are the principal commensal bacteria making up normal biofilm and play a role in resistance to colonization by invading pathogens, keeping the oral cavity clean and moist with oral rinsing should be important for the prevention of oral mucositis. Previous studies have demonstrated that rinsing with lemon-flavored water could promote saliva secretion [30]. In any case, oral rinsing 6 times a day may be sufficient to keep the oral cavity clean and moist.

We conclude that oral rinsing with lemon-flavored water, 
Citation: Kabeya M, Ina K, Yuasa S, Kikuchi F, Tajiri C, et al. (2013) Effects of Oral Rinse using Lemon-Flavored Water with or without Rebamipide on Fluoropyrimidine-Induced Stomatitis. J Integr Oncol 2: 107. doi:10.4172/2329-6771.1000107

supported by multidisciplinary intervention, can successfully attenuate the incidence and severity of fluoropyrimidine-induced stomatitis [31], enabling patients to continue with their chemotherapeutic regimens for extended periods.

\section{References}

1. Sakuramoto S, Sasako M, Yamaguchi T, Kinoshita T, Fuji M, et al. (2007) Adjuvant chemotherapy for gastric cancer with S-1, an oral fluoropyrimidine. N Engl J Med 357: 1810-1820.

2. Koizumi W, Narahara H, Hara T, Takagane A, Akiya T, et al. ( 2008) S-1 plus cisplatin versus S-1 alone for first-line treatment of advanced gastric cancer (SPIRITS trial): a phase III trial. Lancet Oncol 9: 215-221.

3. Doi T, Boku N, Kato K, Komatsu Y, Yamaguchi K, et al. (2010) Phase I/II study of capecitabine plus oxaliplatin (XELOX) plus bevacizumab as first-line therapy in Japanese patients with metastatic colorectal cancer. Jpn J Clin Oncol 40: 913-920.

4. Ajani JA, Rodriguez W, Bodoky G, moiseyenko V, Lichinitser M, et al. (2010) Multicenter phase III comparison of cisplatin/S-1 with cisplatin/infusional fluorouracil in advanced gastric or gastroesophageal adenocarcinoma study: the FLAGS trial. J Clin Oncol 28:1547-1553.

5. Tournigand C, André T, Achille E, Lledi G, Flesh M, et al. (2004) FOLFIRI followed by FOLFOX6 or the reverse sequence in advanced colorectal cancer: a randomized GERCOR study. J Clin Oncol 22: 229-237.

6. Ina K, Furuta R, Kataoka T, Kayukawa S, Yoshida T, et al. (2011) Lentinan prolonged survival in patients with gastric cancer receiving S-1-based chemotherapy. World J Clin Oncol 10: 339-343.

7. McCarthy GM, Awde JD, Ghandi H, Vincent M, Kocha WI (1998) Risk factors associated with mucositis in cancer patients receiving 5-fluorouracil. Oral Oncol 34: $484-490$

8. Sonis ST, Elting LS, Keefe D, Peterson De, Schubert M, et al. (2004) Perspectives on cancer therapy-induced mucosal injury: pathogenesis, measurement, epidemiology, and consequences for patients. Cancer 100: 1995-2025.

9. Cascinu S, Fedeli A, Fedeli SL, Catalano G (1994) Oral cooling (cryotherapy), an effective treatment for the prevention of 5 -fluorouracil-induced stomatitis. Oral Oncology: Eur J Cancer 30B: 234-236.

10. Keefe DM, Schubert MM, Elting LS, Sonis ST, Epstein JB, et al. (2007) Updated clinical practice guidelines for the prevention and treatment of mucositis. Cancer 109: 820-831.

11. Katayama S, Ohshita J, Sugaya K, Hirano M, Momose $Y$, et al. (1998) New medicinal Treatment for severe gingivostomatitis. Int J Mol Med 2: 675-679.

12. Fukuhara K, Terakura M, Katsuragi K, Kodama S, Morisya Y, et al. (2011) The effect of rebamipide and polaprezinc mouthwash and uptake on mucositis induced by chemotherapy. Jpn J Cancer Chemother 38: 2603-2606.

13. Arakawa T, Kobayashi K, Yoshikawa T, Tarnawski A (1998) Rebamipide: overview of its mechanism of action and efficacy in mucosal protection and ulcer healing. Dig Dis Sci 43: 5S-13S.

14. Ishihara K, Komuro Y, Nishiyama N, Yamasaki K, Hotta K (1992) Effect of rebamipide on mucus secretion by endogenous prostaglandin-independent mechanism in rat gastric mucosa. Arzneimittelforschung 42: 1462-1466.

15. Furuta $R$, Ando $T$, Watanabe $O$, et al. (2007) Rebamipide enema therapy as a treatment for patients with active distal ulcerative colitis. J Gastroenterol Hepatol 22: 261-267.

16. Kabeya M, Ina K, Yuasa S, Kikuchi F, Tajiri C, et al. (2011) Pilot study on clinical effects of rebamipide gargle against oral mucositis induced by fluoropyrimidines. Jpn J Cancer Chemother 38: 1133-1136.
17. Ten Cate JM, Imfeld T (1996) Dental erosion, summary. Eur J Oral Sci 104 241-244.

18. Zero DT (1996) Etiology of dental erosion-extrinsic factors. Eur J Oral Sci 104 162-177.

19. Cochrane NJ, Cai F, Yuan Y, Reynolds EC (2009) Erosive potential of beverages sold in Australian schools. Aust Dent J 54: 238-244.

20. Aaronson NK, Ahmedzai S, Bergman B, Bullinger M, Culli A, et al. (1993) The European Organization for research and treatment of cancer QLQ-C30: a quality-of-life instrument for use in international clinical trials in oncology. J Natl Cancer Inst 85: 365-376.

21. Atchison KA, Dolan TA (1990) Development of the geriatric oral health assessment index. J Dent Educ 54: 680-687.

22. Astron AN, Hangejorden O, Skaret E, Trovik TA, Klock KS, et al. (2005) Oral Impact on Daily Performance in Norwegian adults: validity, reliability, and prevalence estimates. Eur J Oral Sci 113: 289-296.

23. Naito M, Ito H, Kanagawa $H$ (2007) QOL scales in the oral health field Comparative evaluation of Japanese version of GOHAI and the OIDP in young subjects. Health Sci and Health Care 7: 24-28.

24. Cassidy J, Clarke S, Díaz-Rubio E, Scheithauer W, Figer A, et al. (2008) Randomized phase III study of capecitabine plus oxaliplatin compared with fluorouracil/folinic acid plus oxaliplatin as first-line therapy for metastatic colorectal cancer. J Clin Oncol 26: 2006-2012.

25. Muro K, Boku N, Shimada Y, Tsuji A, Sameshima S, et al. (2010) Irinotecan plus S-1 (IRIS) versus fluorouracil and folinic acid plus irinotecan (FOLFIRI) as second-line chemotherapy for metastatic colorectal cancer: a randomised phase $2 / 3$ non-inferiority study (FIRIS study). Lancet Oncol 11: 853-860.

26. Rubenstein EB, Peterson DE, Schubert M, Keefi D, McGuire D, et al. (2004) Clinical practice guidelines for the prevention and treatment of cancer therapyinduced oral and gastrointestinal mucositis. Cancer 100 (9 Suppl): 2026-2046.

27. Sonis ST, Peterson DE, McGuire DB, Williams DA (2001) Prevention of mucositis in cancer patients. J Natl Cancer Inst Monogr 29: 1-2

28. Ruescher T, Sodeifi A, Scrivani S, Kaban Lb, Sonis ST (1998) The impact of mucositis on $\alpha$ hemolytic streptococcal infection in patients undergoing autologous bone marrow transplantation for hematological malignancies. Cancer 82: 2275-2281.

29. Yoneda S, Imai S, Hanada N, et al. (2007) Effects of oral care on development of oral mucositis and microorganisms in patients with esophageal cancer. Jpn J Infect Dis 60: 23-28.

30. Kano T, Ohnuma H, Kanda K (2004) Effects of rinsing the mouth with lemonflavored water on saliva secretion and taste threshold. Jpn J Physiol Anthropol 9: 31-37.

31. Soga Y, Sugiura Y, Takahashi K, Nishimoto H, Maeda Y, et al. (2011) Progress of oral care and reduction of oral mucositis-a pilot study in a hematopoietic stem cell transplantation ward. Support Care Cancer 19: 303-307. 